\title{
Un tarif de tonlieu inconnu, institué par le comte de Flandre Thierry d'Alsace (1128-1168) pour le port de Littersuerua, précurseur du port de Damme
}

par Adriaan Verhulst, Thérèse de Hemptinne et Lieve De MEY*

La charte du comte de Flandre Thierry d'Alsace (1128-1168), éditée en annexe au présent article parce qu'elle manque dans l'édition des chartes des comtes de Flandre de la maison d'Alsace, publiée récemment pour la période $1128-1168^{\prime}$, présente un triple intérêt.

Elle fait connaître en premier lieu un port du Zwin, inconnu jusqu'à présent, qui, à l'endroit où en 1180 existera la ville de Damme, a précédé celle-ci. Elle éclaire en deuxième lieu d'une lumière toute nouvelle l'histoire commerciale de la Flandre et des régions avoisinantes. Elle permet enfin d'entrevoir comment était élaborée la politique commerciale du comté de Flandre à cette époque.

L'existence de la charte n'est pas totalement inconnue puisqu'elle est signalée dans l'Inventaire sommaire des Archives du Nord à Lille où elle est conservée dans une copie informe sur deux feuilles de papier du XV' siècle, cousues ensemble. Elle précède dans la même copie une charte de la comtesse Marguerite de Flandre et de son fils Guy du 19 septembre 1269, également publiée pour la première fois

* L'introduction est de la main du premier auteur, tandis que Mesdames Thérèse de Hemptinne, chargée de cours à l'université de Gand, et Lieve De Mey, collaboratrice de la Commission Royale d'Histoirc, ont préparé et assuré l'édition des chartes en annexe. Th. de Hemptinne a collaboré au texte de l'introduction en ce qui concerne la datation. Les documents édités ont été signalés aux auteurs par M. Georges Declercq. chargé de cours à la Vrije Universiteit Brussel, à qui nous devons également les premières indications sur la localisation de Littersuerua. Enfin, M. Jacques De Groote, président du conseil communal du patrimoine culturel de Damme, qui prépare une histoire de la ville de Damme, nous a fait bénéficier de ses recherches dans les archives et de sa connaissance de la région. Il nous a fait part en outre de ses observations critiques et toujours judicieuses à propos de notre introduction.

' Th. DE HEMPTINNE - A. VLRHELST, De oorkonden der graven van Vlaanderen (juli 1/28-september 1/91). II. Uitgave-Band I: Regering van Diederik van de Elzas (juli //28 - 17 jamuari //68). Bruxelles. 1988 (CRH. RECUEIL DES ACTES DES PRINCES BELGES, 6). 
en annexe au présent article ${ }^{2}$. Celle-ci n'est pas une confirmation de la charte de Thierry, mais ordonne aux receveurs du tonlieu de Gravelines d'appliquer à celui-ci le tarif contenu dans la charte de Thierry, à laquelle, selon la charte de Marguerite, cette dernière était attachée. Aucune des deux chartes n'a reçu de la part des historiens l'attention qu'elles méritent, celle de Thierry d'Alsace encore moins que celle de Marguerite, la seule dont le contenu a au moins été analysé brièvement ${ }^{3}$. L'abréviation "Th. » de la suscription, figurant vraisemblablement dans l'original ou dans une copie antérieure à celle du $X V^{e}$ siècle et que cette dernière a résolue erronément par «Thomas ", a pu contribuer à ce manque d'intérêt. L'erreur, déjà remarquée par Godefroy, fut cependant signalée dans l'Inventaire sommaire, car elle n'a pas été répétée dans la copie de la charte de Marguerite, où le nom de Thierry n'a pas été abrégé ${ }^{4}$. La comtesse renvoie explicitement à une charte de Thierry d'Alsace à laquelle la sienne était attachée ( secundum tenorem scripti per bone memorie Theodericum quondam comitis Flandrie, predecessoris nostri, confecti, cui hec nostre presentes littere sunt appense »).

L'attribution par le copiste du $X^{*}$ siècle et peut-être déjà par un copiste antérieur, d'une charte de Thierry d'Alsace au comte de Flandre Thomas de Savoie, deuxième époux de Jeanne de Constantinople, son successeur à presque soixante-dix ans d'intervalle, pourrait s'expliquer par l'invraisemblance du fait que la comtesse Marguerite, ayant succédé à sa sœur Jeanne de Constantinople en 1244, ait fait appliquer au tonlieu de Gravelines un tarif établi environ cent ans plus tôt par un de ses prédécesseurs pour le tonlieu d'un port («portus») dont le nom était apparemment inconnu au copiste du $X V^{*}$ siècle et qui n'était vraisemblablement même plus en usage en 1269 lorsque la comtesse Marguerite ordonna son application à Gravelines. Elle ne cite en tout cas pas le nom de ce port dans sa

- Abbé DehaISNes - J. FInOt, Inventaire sommaire des archives départementales. Nord, Série B, I, 2, Lille, 1906, p. 302 : B1321.

'Th. LuYKX, De grafelijke financiële bestuursinstellingen en het grafelijk patrimonitum in Vlaanderen tijdens de regering van Margareta van Constantinopel (1244-1278). Bruxelles, 1961 (KON. VlaAmSE ACADEMIE VOOR WETENSCHAPPEN, LetTEREN EN SCHONe Kunsten Van Bel.gië. Ki.asse der Letteren. Verhandelingen n" 39), p. 194.

+ ADN Lille, B174, Inventaire Godefroy, II, pp. 569-570, n" 1599 ; DEHAISNES FINOT, Inventaire sommaire, p. 302. n. 1.

Les abréviations « $T(h)$. 》 et « $P h$. » sont assez nombreuses dans les suscriptions des actes des comtes de Flandre conservés en original de cette époque ; Th. DE HEMPTINNE A. VERHulst, De oorkonden der graven van Vlaanderen. II. 1, n" 69, 98, 100, 166, 185. $195,228,247,268$ et 269. 
charte. Dans la copie du $X V^{*}$ siècle de la charte de Thierry il est écrit «Letraswerf».

L'identification de ce toponyme constitue la clef de notre recherche et la solution de plusieurs problèmes qui se posent à propos de ce tonlieu. La graphie qui se trouve dans la copie du $X^{*}$ siècle ( Letraswerf» et même «Le Traswerf») peut être rapprochée, même si elle a été déformée, du nom de «Littersuerua» qu'on rencontre une seule fois dans un autre document du XII ${ }^{*}$ siècle et plus jamais après. L'identification et la localisation qui seront proposées ci-après expliqueront cette apparition éphémère. Le document dans lequel apparaît le nom de «Littersuerua» est une charte de l'évêque Gérard de Tournai de 1163 pour l'abbaye de Saint-Quentin-en-l'Ile, attribuant à celle-ci le personat de l'église d'Oostkerke, au nord-est de Bruges, avec cinq chapelles qui en dépendaient ${ }^{5}$. La charte situe ces cinq chapelles comme suit ${ }^{6}$ : une dans l'île de Wulpen, jadis située devant la

'C. Duvivier, Actes et documents anciens intéressant la Belgique, I, Bruxelles, 1898, pp. 329-330, d’après cartul. Laon H534, fol 8: «personatum quem Dodinus et sui praedecessores olim tenuerunt, nihilominus etiam ipsum altare de Oskerca cum suis appendiciis, videlicet Wulp.s, Lapscura, Murkerca, Vuas, Littersuerua... contradidi$m u s$ » (texte corrigé et établi sur Paris, BN. ms. lat. 12985, f" 5v ). Oostkerke : prov. Fl. Occ., cant. Bruges. Saint-Quentin-en-l'Ile : abbaye bénédictine jadis située dans une île de la Somme à Saint-Quentin, ch-l. arr., dép. Aisne (L. H. CotTineau, Répertoire topobibliographique des abhaỵes et prieurés, II Mâcon 1939, col. 2860). Le patronat de l'église d'Oostkerke appartenait déjà à cette abbaye en 1089 (F. VERCAUTFRen, Actes des comtes de Flandre 1071-1128. Bruxelles, 1938 (CRH, ACTES DES PRINCES BELGES) n" 8. On suppose généralement que l'abbaye l'avait reçu du comte Baudouin V (10351067): voir les articles de l'historien local R. DE KEYSER, dans BIEKORF 60 (1959), pp. 243-246 et ROND de POLdertorens 4 (1962), pp. 61-72 et 20 (1978), pp. 183-185 et 21 (1979) pp. 47-48.

"L'étude de la géographie historique de la région du Zwin au $12^{\circ}-13^{\circ}$ siècle, a été renouvelée, après les travaux toujours utiles pour leurs références de A. DE SMET, Het waterwegennet ten noordoosten van Brugge in de l3e eeuw: RFVUE BELGE DE PHILOLOGIE ET D'HISTOIRE, XII, 1933, pp. 1023-1059 et XIII, 1934, pp. 83-121, réimpression dans Album Antoine De Smet, Bruxelles, 1974, pp. 1-52 (avec résumé français), par M. K. E. GotTschalk, Historische geografie van westelijk Zeeuws-Vlaanderen tot de Sint-Elisabethsvloed van 1404, Assen, 1955, pp. 15-38, 55-61, 110-128 et par A. VERHULST, Middeleeun'se impolderingen en bedijkingen van het Zwin (avec résumé français), Bulletin de la Société belge d'Etudes Géographiques, XXVIII, 1959, pp. 21 54. Un aperçu en français de la géographie historique de la région du $Z$ win au nord de Bruges se trouve dans l'ouvrage de A. Verhulst, Histoire du paysage rural en Flandre, Bruxelles, 1966, pp. 35-50. L'histoire topographique détaillée de la région a fait l'objet d'un très grand nombre d'études d'un érudit local, feu $M$. Coornaert, publiées dans la revue d'histoire locale ROND DE POLDERTORENS, dont une bonne synthèse se trouve dans M. COORnAERT, Bijdrage tot de historische geografie van de streek rondom Brugge, Handelingen van de MaAtschappij voOR Geschiedenis en Oudheidkunde te Gent, nouvelle série, XXI, 1967, pp. 3-33, ainsi que dans le livre M. COORNAERT, Knokke en het Zwin. Tielt, 1974. L'ouvrage le plus récent sur la région est un ouvrage collectif sous la rédaction de F. WEI.vaERT (ed.), 2000 jaar Zwinstreek, Knokke, 1985. 
côte flamande à hauteur de Cadsand (à l'est de Knokke) ', une deuxième à Lapscheure, village situé à ce moment à deux kilomètres au sud de Sluis et à huit kilomètres au nord-est de Damme ${ }^{8}$, une troisième à Moerkerke, à quatre kilomètres à l'est de Damme, une quatrième à Westkapelle, à sept kilomètres au nord de Damme mais de l'autre côté du Zwin, c'est-à-dire à l'ouest de ce chenal, et enfin en dernier lieu, toujours dans l'ordre de l'énumération de la charte, la chapelle de «Littersuerua ». La graphie abâtardie des noms flamands de ces localités, que donnent la plupart des cartulaires de SaintQuentin-en-l'Ile", particulièrement celle du toponyme disparu de «Littersuerua» (écrit «Lesiterruse » dans la plupart de ces cartulaires), rend encore plus difficile l'identification de celui-ci. Il y a heureusement un cartulaire qui donne de ces cinq toponymes une graphie acceptable et qu'on peut considérer comme contemporaine de la charte épiscopale de $1163^{\prime \prime}$.

En réalité celle-ci est une confirmation d'une charte antérieure de l'évêque Baudry de Noyon-Tournai de 1110, confirmée en 1114 par le pape et ayant le même objet, mais dans laquelle l'église d'Oostkerke ne compte à ce moment que quatre au lieu de cinq chapelles dépendantes ". Celle qui manque est précisément la chapelle de «Littersuerua»!

C'est probablement la future paroisse de Bri(e)le dans le sud-ouest de l'île. Celleci, d'une longueur d'environ $16 \mathrm{~km}$ et d'une largeur de $7 \mathrm{~km}$, mais dont la configuration ainsi que l'exacte localisation des paroisses demeurent incertaines, comptait cinq paroisses au total, dont quatre appartenaient à l'abbaye Saint-Bavon de Gand: GotTschalk, Historische geografie. pp. 27-28, 115-116, 120-124; M. CoOrnalert, Het tienderecht in de oorspronkelijke parochie Oostkerke en op het eiland Wulpen met de topografie en de geschiedenis van Wulpen, ROND DE POLDERTORENS 31, 1989, n" 1, pp. 4-35. L'île de Wulpen disparut progressivement dans les flots au cours des $14^{\text {" et }}$ $15^{\prime}$ siècles.

"C'est-à-dire à environ 1200 mètres au nord du village actuel de ce nom, qui fut rebâti à un autre endroit après les inondations stratégiques de la fin du $16^{\circ}$ siècle (1583): VERHULST, Middeleeuwse inpolderingen, p. 27 n. 16 et 31-34, ainsi que la carte p. 55; L. Devlieglier, De Zwinstreek, Tielt, 1970 (KunStPatrimonium van WestVLAANDEREN, 4), p. 105.

"Paris, Archives Nationales, LL 1016 (a" 1386); Laon, Archives dép. Aisne, H534 (18' siècle) ; Paris, Bibl. Nationale, ms. lat. 10116 (a" 1764).

"Paris, Bibl. Nationale, ms. lat. 12985, f" 5 v". Cf. M. Gysseling, Toponymisch Woordenboek van België. Nederland, Luxemburg, Noord-Frankrijk en West-Duitsland (vóór 1226), s.l., 1960, I, p. 625, v' 'Littersuerua'. Voir plus haut note 5.

"Duvivier. Actes, I, pp. 324-325: « in Flandriis super mare, altare de Oskerke cum ecclesia et quatuor capellis: prima de Vulps, quae sita est in maris insula, secunda de Lapscures, tertia de Murkerka, quarta de Vuas ». Confirmation de 1114 dans les mêmes termes par le pape Pascal II, éd. J. Ramackers, Papsturkunden in Frankreich, 4. Picardie, Göttingen, 1942, n"10, p. 77. 
Cette particularité suggère l'hypothèse que celle-ci est plus récente que les quatre autres et que sa fondation doit avoir eu lieu entre $1110 / 1114$ et 1163. On est alors en pleine période de colonisation et de peuplement de la plaine maritime flamande, dont témoignent les noms en -kerke et en -kapelle '?. Ceci est particulièrement vrai de la région d'Oostkerke au nord de Bruges. La grande inondation de 1134 qui a touché l'ensemble des régions autour de l'embouchure de l'Escaut y a réactivé plusieurs chenaux d'inondations antérieures. Elle, ou l'une ou l'autre des inondations qui l'ont suivie dans les années soixante et soixante-dix du XII ${ }^{\circ}$ siècle, a creusé, partiellement dans leur lit, le grand chenal du Zwin, accessible à des navires de mer ${ }^{13}$. C'est sur le bord d'un de ces chenaux que doit être cherché, à notre avis, peut-être même antérieurement à l'inondation de 1134, le port de "Littersuerua », car il s'agit bien d'un port d'après le texte de la charte de Thierry d'Alsace («portus»). Le premier argument en faveur de cette hypothèse est la situation géographique de la chapelle de ce port, vis-à-vis de l'église d'Oostkerke dont elle dépendait et visà-vis des autres chapelles qui en dépendaient selon les chartes épiscopales de 1110 et 1163 . La paroisse d'Oostkerke, dont la première mention date de 1089, était une des plus étendues au nord de Bruges. Située à l'est de la paroisse plus ancienne de Dudzele et peut-être issue de celle-ci, elle occupait toute la moitié est du territoire entre Bruges, la côte et Aardenburg, c'est-à-dire également le territoire sur la rive droite du Zwin. Ce grand chenal du Zwin n'existait donc visiblement pas encore au moment de la formation de la paroisse et même lors de la fondation des chapelles dépendantes situées à l'est du Zwin, dont l'église-mère se trouvait sur la rive gauche du Zwin ". Les chapelles de Westkapelle et dans l'île de Wulpen étaient situées au nord

12 Aperçu général dans Verhulst, Histoire du paysage rural, pp. 25-58.

1 Ibidem, pp. 41-42; A. VERHULST, L'évolution géographique de la plaine maritime flamande au moven âge, REVUE DE L'UNIVERSITÉ DE BRUXELLES, oct. 1962-janvier 1963, n' 1-2, pp. 89-106 ; S. LEBECQ, L'homme au péril de l'eau dans les plaines littorales des anciens Pays-Bas au début du moven âge, dans: B. BENNASSAR (éd.), Les catastrophes naturelles dans l'Europe médiévale et moderne. Toulouse, 1996, (FLARAN XV), pp. 2742. spéc. pp. 34-35. Les sources concernant les inondations de 1134 et de celles qui l'ont suivie, plus particulièrement en 1164 et en 1170, ont été rassemblées et soumises à une étude critique sévère par M. K. E. GotTSCHALK, Stormvloeden en rivieroverstromingen in Nederland, I, Assen, 1971, pp. 59-119 (avec de longs résumés en anglais).

${ }^{14}$ A. VERHULST, Les biens et revenus du chapitre Saint-Donatien de Bruges en 1089, dans: E. MORNET (éd.), Campagnes médiévales: l'homme et son espace. Études offertes à Robert Fossier, Paris, 1995, pp. 513-531; COORNAERT. Het tienderecht in de oorspronkelijke parochie Oostkerke: VERHULST, Middeleeuwse inpolderingen, pp. 2, 32 ; Devliegher. Zwinstreek, pp. 147 suiv. et les travaux cités plus haut note 6. 
d'Oostkerke et à l'ouest du Zwin, celle de Lapscheure à l'est et celle de Moerkerke au sud-est d'Oostkerke, toutes les deux sur la rive droite du Zwin. Il ne reste donc pour la nouvelle et cinquième chapelle, celle de "Littersuerua», que la partie du territoire au sud-ouest d'Oostkerke comprise plus tard, au XIII ${ }^{\circ}$ siècle, dans la paroisse de SainteCatherine-lez-Damme, à l'est et au sud-est de Damme ${ }^{15}$.

Pour vérifier cette hypothèse, la situation ecclésiastique de la région au bas moyen âge et plus particulièrement les documents concernant les droits ecclésiastiques de l'abbaye de Saint-Quentin-en-l'Ile, notamment la localisation des dîmes qu'elle possédait dans la paroisse d'Oostkerke et dans toutes les paroisses qui en ont été détachées, devraient pouvoir nous aider ${ }^{16}$. Seulement, comme on l'a déjà dit, le toponyme «Littersuerua» ne se retrouve plus jamais dans aucun document, ni de l'abbaye ni d'aucune autre institution, après son éphémère apparition dans la charte épiscopale de 1163 et, sous une forme abâtardie, dans la copie de la charte de Thierry d'Alsace dont on essaiera de démontrer plus loin qu'elle date des années 1159-1168 et même probablement de peu de temps avant 1163. Cette disparition du toponyme est d'autant plus curieuse que de nombreux toponymes en -werve se rencontrent en Flandre maritime, notamment dans la région qui nous concerne, pendant la deuxième moitié du XII siècle et qu'ils ont été conservés dans des documents d'une époque postérieure. Ils sont même particulièrement nombreux aux environs d'Aardenburg, à une bonne dizaine de kilomètres à l'est de Damme, ainsi qu'aux environs de cette dernière ville elle-même ${ }^{17}$.

15 Nous suivons ici le raisonnement de CoORnAERT, Het tienderecht, p. 17.

"Ces documents sont inventoriés et analysés dans B. Augustyn - H. Rombalt M. VANDERMAESEN. Bronnen voor de agrarische geschiedenis van het middeleeun'se graafschap Vlaanderen, II, Gand, 1990 (CENTRE BELGE D'HISTOIRE RURALE. Publication, n" 95), pp. 25-26, 128-129. Ils ont été mis à contribution par VerHulst, Middeleeuwise inpolderingen. Sur les origines et la consistance des biens de l'abbaye de Saint-Quentin-en-l'Ile dans la région: J. OPDEDRINCK, Oostkerke en de abdij van SintQuintens-ten-Eilande, BIEKORF 26 (1920), pp. 211-225; R. DE KEYSER. Wanneer bekwam de Sint-Quintensabdij bezittingen te Oostkerke?., BIEKORF 60 (1959), pp. 243-246 et les publications du même auteur citées plus haut n. 5 ; COORNAERT, Het tienderecht.

Aux environs d'Aardenburg: Hannekenswerve (1169), Monnikenwerve (1174), Oudenwerve (1189/90), Suedekinwerf (1197): GotTschalk, Historische geografie, index, sub v"; aux environs de Damme: Romboutswerve (DE SMET, Waterwegennet, p. 28); à Dudzele: Blevinswerf, Buggherswerf, Houtwerf, Outvaartswerf, Ververildenwerf, Zuudwerve (M. CoORnAERT, Dudzele en Sint-Lenaart, Dudzele, 1985, registre alphabétique, pp. 501-621, sub $\left.\mathrm{v}^{\prime \prime}\right)$; voir aussi C. TAVERNIER-VEREECKEN, Gentse Naamkunde van ca. 1000 tot 1253 (s.I., 1968), p. 622. 
La conclusion s'impose donc que ce toponyme a disparu et même assez tôt, à la fin du $\mathrm{XII}^{\star}$ ou au début du XIII' siècle, lorsque les documents deviennent plus nombreux mais ne le mentionnent plus. Cette disparition peut avoir été la conséquence d'un changement topographique, ce qui n'étonnerait guère dans une région comme celle du Zwin, soumise encore longtemps après à l'influence de la mer et où les endiguements ont continué jusqu'à la fin du XIII' siècle. Il est possible aussi que ce centre de peuplement pourvu d'une chapelle et qui portait le nom de «Littersuerua », ait été délaissé au profit d'une localité toute proche, située pratiquement au même endroit mais appelée d'un nouveau nom qui aurait supplanté l'ancien. Nous croyons fermement à la seconde de ces possibilités. Un endroit d'un nom en -werve a, en effet, généralement bien résisté à d'éventuelles inondations, le nom ayant désigné généralement une localité, une ferme ou même une parcelle dans les régions maritimes des anciens Pays-Bas située sur une hauteur naturelle mais parfois aussi artificielle ${ }^{18}$. La deuxième possibilité, par contre, peut être rendue vraisemblable à l'aide d'une étude de l'évolution topographique de Damme et de ses environs au XIII" siècle.

L'histoire topographique de Damme est bien connue ${ }^{19}$. La ville doit son origine et son nom à la construction vraisemblablement dans les années soixante-dix du $\mathrm{XII}^{\mathrm{c}}$ siècle, comme il sera démontré ci-après, d'une digue transversale à l'endroit où le grand chenal du Zwin devenait trop étroit et trop peu profond pour la navigation. Cette digue barrait le Zwin à hauteur du futur hôtel de ville de Damme, derrière lequel le chenal s'élargissait en direction de la mer. Sa construction s'accompagna d'autres travaux, notamment le creusement, à partir du flanc sud de la digue, d'un canal en direction de Bruges, appelé Reie, relié au Zwin par une écluse dans la digue (appelée plus tard la "grande speye »), à côté de l'hôtel de ville. D'autres grands travaux furent la construction de quais aux environs immédiats de l'écluse, notamment sur la Reie. C'était la grande nouveauté qu'offrait le port de Damme par rapport aux autres ports du Zwin qui étaient et sont restés, à l'exception de L'Ecluse (Sluis), des ports de marée. Egalement à Damme même, immédiatement au nord de la digue, le Zwin n'était

"* Tavernier-Vereecken, Gentse Naamkunde, pp. 549-550.

1" DE SMET, Waterwegennet et ID., L'origine des ports du Zwin Damme, Mude, Monikerede, Hoeke et Sluis, dans: Études d'Histoire dédiées à la mémoire de Henri Pirenne par ses élèves, Bruxelles, 1937, pp. 125-141, spéc. pp. 127-132; L. Devl.iegher, Damme, Tielt, 1971 (KunstPatrimonium van West-VlaAanderen 5); J. TERMOTE, Het ontstaan en de stadsontwikkeling van Damme, dans : WELvaERT (éd.), 2000 jaar Zwinstreek. pp. 102-111. 
pas encore pourvu de quais au début du XIII' siècle. Ces différents grands travaux ont vraisemblablement été entrepris et financés par la ville de Bruges en collaboration avec le comte de Flandre. Celui-ci a sans doute prêté son concours en facilitant la mise à la disposition de la ville de Bruges des terrains nécessaires, notamment par le moyen de l'expropriation ${ }^{20}$. La ville de Bruges devint ainsi maître de l'infrastructure ${ }^{21}$. Entretemps Damme doit avoir reçu du comte de Flandre son propre banc d'échevins et donc le statut de ville. En 1180, en effet, lorsque le nom de «Dam » apparaît pour la première fois, les bourgeois («burgenses ») de Damme, habitant à l'intérieur de l'échevinage («infra scabinatum eiusdem ville manentibus») reçurent du comte Philippe d'Alsace d'importants privilèges commerciaux, notamment l'exemption de tonlieu dans tout le comté, comme l'avaient déjà reçu du même comte en 1163 les villes nouvelles de Nieuport et de Gravelines, fondées par lui également comme ports de mer ${ }^{22}$.

Le décalage entre les dates de 1163 (apparition de «Littersuerua» et date de fondation des ports nouveaux de Nieuport et Gravelines) et 1180 (privilèges commerciaux pour Damme et première mention du nom de la ville) pose cependant un problème. Bien que le chenal du Zwin soit généralement considéré comme ayant été formé déjà en $1134^{23}$, les travaux pour l'aménagement d'un port à Damme n'ont vraisemblablement commencé que dans les années soixante-dix. C'est ce que suggère une charte comtale de 1174 concernant une expropriation sur les bords du Zwin près de Damme par le comte, en vue de la

20) Voir ci-après note 24.

"Cette situation se réflète au 13' siècle dans les dépenses nombreuses et coûteuses que la ville de Bruges a dû faire pour l'entretien de cette infrastructure: C. WYFFELs, De rekeningen van de stad Brugge (1280-1319). Eerste deel (1280-1302), 1, Bruxelles, CRH, 1965 et 2, Indices, Bruxelles, 1971 , v. Damme.

$\because \mathrm{H}$. VAN WERVEKE, De economische politiek van Filips van de Elzas (1157-68 tot 1191) (avec résumé français), Bruxelles, 1952, (MEUtdELINGin VAN dE KuN. VLAAMSE ACademie voor Wetenschappen, Letteren en Schone Kunsten van België, XIV, 3) ; A. VERHULST, Initiative comtale et développement économique en Flandre au XII siècle; le rôle de Thiern et de Philippe d'Alsace (II28-1191), dans Miscellanea Mediaevalia in memoriam Jan Frederik Niermeyer, Groningen, 1967, pp. 227-240; R. DEgRYSE, Oude en nieuwe havens van het IJzerbekken in de middeleeuwen, Handelingen van het Genootschap «SociÉtÉ d'Émulation 》 TE BRUgGe 84, 1947, pp. 2-10 (conc. Nieuport); A. Verhulst, Un exemple de la politique économique de Philippe d'Alsace : la fondation de Gravelines (1163), CAHIERS DE CIVILISATION MÉdiéVAlE, 10, 1967, pp. 15-28; A. DeRville, Les origines de Gravelines et de Calais, Revue Du Nord, 56, 1984, pp. 1051-1069; R. Degryse, Nieuwpoort tot omstreeks 1302, Nieuwpoort, 1987.

${ }^{27}$ Voir les travaux cités plus haut note 13 et ci-après note 25. 
construction d'une digue ${ }^{24}$ ainsi que la situation pédologique au sud de Damme ${ }^{25}$. D'autre part on a toujours considéré jusqu'à présent, 'et avec raison, la construction de la digue transversale devant l'endroit où s'élèvera plus tard l'hôtel de ville et celle de l'écluse, comme le point de départ de l'expansion de la ville. Cette extension aurait eu lieu du nord au sud, c'est-à-dire en s'éloignant de la digue à travers le Zwin et du canal de la Reie. On ne s'est toutefois pas demandé si les possibilités ouvertes à la navigation par la formation du Zwin à partir de 1134 n'auraient pas été utilisées pendant les décennies précédant la construction de la digue et des quais. L'existence, au plus tard vers 1163-1168, d'un port à "Littersuerua», sans doute, comme nous avons essayé de le démontrer, un port de marée sur les bords du Zwin aux environs immédiats de Damme et ayant précédé celui autour de la digue transversale à Damme même, rend une telle supposition très vraisemblable. Dans ce cas le problème de localiser le port de «Littersuerua » aussi exactement que possible, s'impose.

Peut-être la petite église de Sainte-Catherine-lez-Damme peut-elle nous aider sur ce point ${ }^{26}$. Cette église, disparue depuis lors, était située au sud-est de Damme, aux portes mêmes de la ville, juste en dehors des fortifications de la fin du XIV'-début du $X^{*}$ siècle, bien qu'elle ait été comprise dans le premier rempart urbain, datant des années

:Charte originale de Philippe d'Alsace de 1174 (n"371 dans l'édition future de Th. DF. HEMPTINNE - A. Verhulst, De Oorkonden der graven van Vlaanderen (Juli 1128-September 1191). II. Uitgave-Band II : Regering van Filips van de Elzas) : "quoniam pro terra Sancti Donatiani quam in faciendo fossato occupari iussi in parrochia de Ostkerca XL solidos per singulos annos eidem ecclesie me solviturum constitui ». A rapprocher de l'apparition (récurrente) de cette dépense dans le plus ancien compte général conservé du comté de Flandre, datant de 1187, dans les termes suivants: "Sancto Donatiano pro terra cambita in Swen 2 l. » (A. Verhulst - M. GysSELING, Le Compte Général de 1187, connu sous le nom de "Gros Brief » et les institutions financières du comté de Flandre au XII siècle. Bruxelles, 1962, (CRH in-8"), pp. 134, 177. Les propriétés du chapitre Saint-Donatien de Bruges entre Bruges et Damme («up sprocfs ») aboutissaient au canal de la Reie, creusé pour relier par eau Bruges à Damme, vraisemblablement dans les années soixante-dix : De. SMET, WATERWEGENNET, p. 84 n. 4 (p. $28 \mathrm{n} .134$ de la réimpression).

2. M. K. E. GotTSChalk, Stormvloeden en rivieroverstromingen in Nederland, I. De periode vóór 1400. Assen, 1971, pp. 116-119 (résumé en anglais) fait état d'une couche d'argile de $50 \mathrm{~cm}$. d'épaisseur déposée au sud de la digue de Damme, vraisemblablement et principalement par la grande inondation de 1134. Cette épaisseur correspond. selon les pédologues, à environ cinquante ans de submersion, dont la fin correspond donc à peu près à l'époque de la construction de la digue transversale qui a donné son nom à la ville de Damme.

${ }^{2 h}$ L'hypothèse qui suit nous a été suggérée par l'identification de 'Littersuerua' avec Sainte-Catherine-lez-Damme, proposée, toutefois sans argumentation, par COORNAERT, Tienderecht, p. 17. 
1297-1298 ${ }^{27}$. Elle se situait dans le prolongement de la rue principale de la ville, la Kerkstraat. Celle-ci prenait son départ dans le nord de la ville, sur la place devant l'hôtel de ville où elle s'identifiait avec la digue transversale dans le Zwin, qu'elle prolongeait vers le sud-sudest. Dans ce dernier secteur de la ville l'église Sainte-Catherine se trouvait donc juste à l'autre bout de l'axe principal que la digue transversale et l'hôtel de ville. Comme l'église urbaine principale, dédiée à Notre-Dame, se situe dans la Kerkstraat, à peu près à mi-chemin entre l'hôtel de ville et l'église Sainte-Catherine, cette dernière a été considérée jusqu'à présent comme le témoin de l'expansion maximale de la ville médiévale ${ }^{2 x}$.

Nous doutons sérieusement du bien-fondé de cette interprétation du développement topographique de Damme. La paroisse de SainteCatherine, en effet, était au bas moyen âge avant tout une paroisse rurale, dont le ressort s'étendait sur un certain nombre de polders à l'est et au nord-est de Damme, le long du Zwin (Amelispolder, Vijf Polders et une partie du Grote Nieuwe Polder). Ceux-ci ont été gagnés sur le Zwin lui-même par des endiguements pendant la deuxième moitié du XIIIe siècle, à partir du Sluisse Dijk, qui depuis 1228 ou même plus tôt, jusqu'aux endiguements de la deuxième moitié du XIIIe siècle a été la principale digue sur la rive droite du Zwin au nord de Damme ${ }^{29}$. L'église Sainte-Catherine se trouvait derrière cette digue, qui la protégeait des eaux du Zwin. Elle était d'ailleurs située sur la même élévation naturelle que la Kerkstraat. Au bas moyen âge elle s'y trouvait pratiquement seule, sans maisons autour d'elle, comme le montre encore vers 1570 la célèbre carte du Franc de Bruges de Pierre

\footnotetext{
${ }^{27}$ Devliegher, Damme, p. 21 et la reproduction, à la fin de l'ouvrage, du plan de Damme de Jacques de Deventer (fig. $\mathrm{n}^{\prime \prime} 2$ ) et d'un détail de la carte des wateringues «van den Broucke en Moerkerke Zuid over de Leie» de Pierre Pourbus (1574) (fig. $\mathrm{n}^{\prime \prime} 3$ ) et de «Romboutswerve », reproduites dans P. Huvenne, Pieter Pourbus, meesterschilder, 1524-1584, Bruges, 1984, respectivement ni* 42 el 43. Une meilleure reproduction de la carte de la wateringue de «Romboutswerve» se trouve dans MolENECHO's. VlaAms Tundchrift voor molinologie, 25, 1997, pp. 84-85 (article de J. De Groote. De eerste (beschreven) ros-en windwatermolens in Vlaanderen). Nous remercions cet auteur de nous avoir fourni cette référence ainsi que des précisions sur l'ancienne topographie de Damme. Voir aussi Termote, Ontstaan van Damme, pp. 106-109 et fig. 2, 4 et surtout 6 et 7 .

2* Termote, Ontstaan van Damme, pp. 107-108.

29 AuguSTIN - ROMBAUT - VANDERMAESEN, Bronnen agrarische geschiedenis, pp. 25-26; OPDEDRINCK, Oostkerke en Sint-Quintens-ten-Eilande, p. 224; VERHULST, Inpolderingen, pp. 40-41 et p. 46 ; G. Adriaensens - D. Vogelaers - M. STrobBe, De historische polders tussen Damme en Sluis, WestVlaAmSE ARChafologica 3, 1987, I, pp. 19-28, spéc. pp. 23-24.
} 
Pourbus et en 1578 sa carte de la wateringue de "Romboutswerve ", ainsi qu'un registre aux dîmes et autres revenus de l'abbaye de SaintQuentin-en-l'Ile vers $1400{ }^{30}$. On doit donc supposer que, sauf l'église, les habitations au centre de la paroisse aient disparu au cours des XIII et $\mathrm{XIV}^{\mathrm{c}}$ siècles, car autrement on se demande ce qui a pu justifier, aux yeux de l'abbaye de Saint-Quentin-en-l'Ile, l'érection d'une église à cet endroit vers le milieu du XII' siècle, avant l'existence de Damme, et son élévation au rang de paroisse, sans doute au XIII', à un moment que son ressort paroissial ne se composait pas encore des polders dessusdits. Il est, en effet, invraisemblable que la paroisse ait été érigée en fonction de ces endiguements de la deuxième moitié du XIII" siècle ", alors que son centre se trouvait dans la ville même de Damme, comme il ressort du tracé des fortifications urbaines de la fin du XIII' siècle, bien que la paroisse de Sainte-Catherine n'ait jamais fait partie de l'échevinage urbain mais ressortissait sous le métier d'Oostkerke.

Il n'y a, à notre avis, qu'une seule solution aux problèmes que pose l'existence, à cet endroit, d'une église isolée: c'est de supposer qu'elle puisse être identifiée avec la chapelle de «Littersuerua», confirmée comme dépendance de l'église d'Oostkerke à l'abbaye de SaintQuentin-en-l'Ile par l'évêque de Tournai en 1163. On pourrait ensuite supposer que cette chapelle, érigée entre 1114 et 1163, ait été destinée en premier lieu à desservir un port de marée qui s'était développé sur les rives du Zwin au nord-est de la future Kerkstraat à Damme, avant l'existence de la ville proprement dite, c'est-à-dire avant les travaux d'aménagement d'un port pourvu de quais plus au nord, à l'autre bout de la Kerkstraat, lorsque, pendant les années soixante-dix du $\mathrm{XII}^{*}$ siècle, le canal de la Reie fut creusé pour assurer la liaison par eau du nouveau port avec Bruges. Le développement du nouveau port aux environs du futur hôtel de ville, en attirant les navires amarrant jusqu'alors sur l'estran du port de marée de "Littersuerua", aura déplacé le point de gravité de l'agglomération naissante vers cet endroit et rendu excentrique la chapelle de "Littersuerua", remplacée, à mi-chemin entre celle-ci et le nouveau port, par l'église NotreDame au début du XIII' siècle ${ }^{32}$. Le nom même de la chapelle et du port naturel qu'elle desservait aura été éclipsé par celui du nouveau port de Damme et sera tombé très vite dans l'oubli. C'est un phénomène qu'on observe également dans deux ports nouveaux, fondés par

\footnotetext{
"Voir ci-devant notes 27 et 29.

"Voir ci-devant note 27.

"Devliegher, Damme, pp. 56 et suiv.
} 
le comte de Flandre Philippe d'Alsace en 1163 dans des situations analogues sur la côte flamande, peu de temps avant l'existence de Damme: Nieuport à l'embouchure de l'Yser, dont le nom éclipsa celui de Sandeshoved et Gravelines, à l'embouchure de l'Aa, à l'ouest de Dunkerque, fondé à côté de l'ancien port de pêche de SaintWillibrord, qui subsista comme paroisse mais dont le tonlieu fut transféré dans le nouveau port ${ }^{33}$.

En tenant compte de la probabilité très grande de l'identification proposée du port de «Littersuerua » avec le port de marée du Zwin, qui au plus tard vers 1163 aurait précédé le port aménagé de la ville de Damme, pratiquement à l'endroit même où celle-ci naîtra comme ville nouvelle peu avant 1180 , il faut maintenant étudier la charte ellemême dans laquelle Thierry d'Alsace, à une date inconnue, a fait mettre par écrit le tarif du tonlieu du port de «Littersuerua».

Comme c'est très souvent le cas avec de pareils documents, le tarif a été mis par écrit à l'occasion d'une plainte, émanant en l'occurrence de marchands de Cologne. Nous reviendrons plus loin sur la très grande signification économique de leur présence sur les rives du Zwin à une date qu'on essayera d'établir et qui peut être considérée comme précoce du point de vue de l'histoire économique du comté de Flandre.

Dans la copie du $X V^{c}$ siècle qui, rappelons-le, est seule à faire connaître la charte du comte Thierry d'Alsace, celle-ci n'est pas datée. Tout le protocole final manque d'ailleurs, ce qui ne semble pas dû à la copie du $X^{*}$ siècle, ni à la charte de la comtesse Marguerite de 1269, à laquelle la charte de Thierry, qui suit immédiatement celle-ci dans la même copie du $X V^{*}$ siècle, était jadis attachée. La charte de Marguerite n'était pas, en effet, une simple confirmation ou un vidimus de celle de son prédécesseur. Elle se réfère à la charte de Thierry comme "pièce annexée », dans le but de faire appliquer le tarif de «Littersuerua » au tonlieu de Gravelines. Il est donc possible que l'original ou la copie de la charte de Thierry qu'accompagnait celle de Marguerite, n'ait pas eu de protocole final ayant éventuellement compris une date. Cela n'étonnerait pas dans un tarif de tonlieu, même si celui de "Littersuerua» commence par un protocole en bonne et due forme, avec préambule et suscription. Les formes diplomatiques des actes dans lesquels étaient insérés des tarifs de tonlieu variaient à l'infini et se caractérisent par une très grande liberté ${ }^{.4}$. Il

\footnotetext{
${ }^{33}$ Voir plus haut note 22.

is G. DESPY, Les tarifs de tonlieux, Turnhout, 1976 (TyPOLOGIE DES SOURCES DL; MOYEN AGE OCCIDENTAL 19), pp. 19-21.
} 
n'est donc pas totalement exclu qu'il y ait eu un eschatocole dans l'original.

A propos de cet original plusieurs autres problèmes se posent d'ailleurs. Si l'on accepte notre hypothèse concernant l'effacement du port de «Littersuerua » au profit de celui de Damme et si l'on tient compte du fait que le tarif du tonlieu de Damme a été mis par écrit au plus tard en mai 1252 par la comtesse Marguerite et son fils Guy ${ }^{35}$, on s'explique que le tarif de l'ancien tonlieu de "Littersuerua " et plus particulièrement la charte dans laquelle il était consigné, aient perdu à ce moment et peut-être depuis un certain temps déjà, leur signification et leur valeur. Marguerite pouvait donc, en 1269, utiliser en toute liberté la charte de Thierry d'Alsace et même la donner à un tiers. Disposait-elle de l'original lorsqu'elle y attacha sa propre charte afin de donner à l'ancien tarif une nouvelle fonction dans le cadre du tonlieu de Gravelines? En restait-il plusieurs expéditions, dont par exemple une conservée dans les archives comtales? On pourrait le croire à la lumière d'un certain nombre de ressemblances avec le tarif du tonlieu de Damme de 1252, mis par écrit par la même comtesse Marguerite à la demande des marchands de la Hanse allemande ${ }^{36}$.

Il n'en reste pas moins que la procédure suivie par la comtesse Marguerite en 1269 est étrange. Plus d'un siècle après l'établissement du tarif du tonlieu de "Littersuerua »- dont on ne sait pas pendant combien de temps il a été en vigueur avant d'être remplacé par celui du tonlieu de Damme mis par écrit au plus tard en 1252 - la comtesse Marguerite donna la charte dans lequel le tarif de «Littersuerua » était établi et qui visiblement avait perdu sa valeur juridique, au receveur du tonlieu de Gravelines afin de s'y conformer. La comtesse précise que si des marchandises arrivent à Gravelines qui ne sont pas mentionnées dans la charte de son prédécesseur, elles doivent néanmoins être taxées selon les modalités ( «secundum valorem et quantitatem ») contenues dans celle-ci. Bien que le procédé de la remise d'une charte ayant perdu sa valeur juridique ait été inhabituel, à ce que nous sachions, il est néanmoins vrai qu'en général les tarifs proprement dits changeaient peu d'un siècle à l'autre, même si du XII' au $\mathrm{XIII}^{*}$ siècle, par exemple, la valeur de la monnaie flamande, par suite de l'inflation, avait beaucoup diminué ${ }^{37}$. D'anciens tarifs, bien que

" K. Höhl.baum - K. KunzF - W. Stein, Hansisches Urkundenbuch. I, (Halle, 1876), n" 432, pp. 143-147.

"Voir plus loin pp. 164-165.

"C. WYFFELS, Contribution à l'histoire monétaire de la Flandre au XIIT siècle, ReVue belge de PHILOL.ogif ET D'HISTOIRE, 45, 3-4, 1967, pp. 1113-1141. 
très souvent augmentés de dispositions supplémentaires et de marchandises nouvelles, pouvaient ainsi rester en vigueur pendant plusieurs siècles, mais - différence notable avec le cas qui nous occupe - dans le cadre du même tonlieu ${ }^{3 x}$. Pourquoi la comtesse Marguerite a-t-elle en 1269 imposé à Gravelines, où un tonlieu existait depuis le début du XII ${ }^{\circ}$ siècle ${ }^{39}$ et sans doute aussi un tarif dont nous ignorons tout avant la fin du moyen âge, l'ancien tarif de «Littersuerua»? Le fait que celui-ci, par suite du développement de Damme, soit devenu disponible à d'autres fins, n'explique évidemment pas tout, ni le fait que Gravelines était également un port de mer.

Il est bien connu que la comtesse Marguerite, dont les finances étaient en mauvais état, s'est occupée énergiquement de ses ressources financières et notamment de ses tonlieux, au sujet desquels elle est souvent entrée en conflit avec des marchands étrangers "4t. A Gravelines la situation se compliquait encore du fait que cet ancien avant-port de Saint-Omer, dominé par cette dernière ville comme Damme l'était par Bruges ${ }^{* 1}$, est resté flamand après l'annexion définitive de l'Artois et de la ville de Saint-Omer par le roi de France en 1212. D'autre part la comtesse Marguerite a essayé en 1262 de détourner vers Gravelines les marchands de La Rochelle, de Saint-Jeand'Angely, de Niort, du Poitou, de la Gascogne et d'autres régions viticoles du Sud-Ouest de la France, au détriment de Damme ${ }^{42}$. Nous avons l'impression, sans avoir étudié en détail cette histoire, que la comtesse Marguerite, dans les années soixante du XIII ${ }^{\circ}$ siècle, s'est particulièrement occupée de Gravelines. Le fait qu'après la perte de Saint-Omer, la ville flamande de Dixmude soit devenue le chef-desens de Gravelines ${ }^{43}$, peut également avoir joué un rôle dans l'application à Gravelines de l'ancien tarif du tonlieu de «Littersuerua ». Celui-ci, en effet, comme le texte de la charte de Thierry d'Alsace le dit explicitement, a été établi par ce comte sur le modèle du tonlieu de Dixmude avec la participation active des échevins de cette ville. Peutêtre s'en est-on souvenu à Dixmude cent ans plus tard, lorsque la ville

${ }^{*}$ DEsPy, Tarifs de tonlieux, p. 35 .

2" Voir plus loin notes 51 et 52 .

د") LUYKX, Grafelijke financiële bestuursinstellingen, pp. 48-52.

+Voir plus haut note 22 et plus particulièrement Verhulst, Initiative Comtale, p. 230 n. 11.

*H. PIRENNE, Un grand commerce d'exportation au moyen age: les vins de France. ANNALES D’hISTOIRE ÉCONOMIQLie et SOCIAI.F. 5.1933 ; réimpression dans ID.. Histoire économique de l'Occident Médiéval, Bruges. 1951. pp. 600-601.

"Lille, ADN. B 219, f "196v". 
était devenue le chef-de-sens de Gravelines. Peut-être même avait-on conservé à Dixmude un exemplaire du tarif de «Littersuerua»?

Le rôle de Dixmude dans l'établissement du tarif du tonlieu de «Littersuerua » par Thierry d'Alsace environ cent ans plus tôt, mérite, par conséquent, un examen particulier, également à d'autres égards. Malheureusement on sait très peu du tonlieu de Dixmude, dont l'origine remonte probablement à la fin du $\mathrm{XI}^{*}$ siècle, peu de temps après la première mention, en 1089, d'une chapelle à cet endroit. Bientôt détachée de la paroisse rurale d'Esen, l'érection de cette chapelle signifie le début de l'urbanisation de Dixmude ". On peut attribuer celle-ci à sa situation géographique sur l'Yser et au développement de la ville d'Ypres dont Dixmude devint à ce moment l'avant-port ${ }^{45}$. Comme son nom l'indique le développement urbain de Dixmude devint possible à partir de l'endiguement («dic »= digue) des rives de l'Yser. C'est à quelques kilomètres en amont de Dixmude que cette rivière entre dans une large vallée à travers laquelle au cours des siècles précédents elle avait dû chercher son chemin vers la mer. Elle l'avait fait par plusieurs bras jusqu'à ce que dans la première moitié du XIe siècle, quelques grandes inondations marines et les endiguements qui les ont suivies tout au long des $\mathrm{XI}^{*}$ et $\mathrm{XII}^{*}$ siècles firent du cours actuel entre Dixmude et Nieuport, le lit définitif de l'Yser ${ }^{46}$. La fondation en 1163 de Nieuport sur la côte de la Mer du Nord, près de l'embouchure de l'Yser, marque la fin de l'endiguement de cette rivière. Jusqu'à ce moment Dixmude avait été le port de mer principal du comté de Flandre, car le Zwin ne s'est formé que vers 1134 et Bruges n'avait plus à cette époque la communication par eau avec la mer qu'elle avait probablement eue au IX" et peut-être encore dans la première moitié du $\mathrm{XI}^{\mathrm{c}}$ siècle et à laquelle la ville doit sans doute son origine ${ }^{47}$. Saint-Omer d'autre part a dû attendre la canalisation de l'Aa vers le milieu du XII" siècle pour avoir un accès direct à la mer ${ }^{4 \times}$. Son

${ }^{*}$ M. Gyssel. 1950, p. 297 : «Aecclesia de Hesna, cum capellis sibi attinentibus, Dicasmutha et Clare ».

". Degryse, Oude en nieuwe havens, pp. 2-10.

"A. Verhulst, Landschap en landiouw' in middeleeuw's Vlaanderen, Bruxelles, 1995, pp. 13, 20-26.

${ }^{17}$ Voir plus haut les travaux cités notes 6 et 13, ainsi que M. RYCKAERT, Les origines et l'histoire ancienne de Bruges : l'état de la question et quelques données nouvelles, dans J. M. Duvosquel - E. Thoen (éds.), Peasants and Townsmen in medieval Europe. Studia in honorem Adriaan Verhul.st, Gand, 1995, pp. 117-134.

${ }^{\star *}$ A. Derville, Saint-Omer des origines au début du 14' siècle, Lille, 1995, pp. 6973. 
développement urbain jusqu'à ce moment n'a pas pu donner au port de pêche de Saint-Willibrord, à l'embouchure de l'Aa et à côté duquel sera fondée en 1163 la ville de Gravelines, la même impulsion que l'ascension fulgurante d'Ypres a vraisemblablement donnée au développement économique de Dixmude à la fin du $\mathrm{XI}^{*}$ siècle et pendant les premières décennies du XII ${ }^{49}$. Dixmude, en effet, devint, vraisemblablement au cours du XII siècle, un des principaux membres, après Bruges et Ypres, de la Hanse flamande de Londres ${ }^{50}$.

Le tonlieu de Dixmude est mentionné pour la première fois dans la grande 'keure' du comte de Flandre Guillaume Cliton pour la ville de Saint-Omer en 1127. Le comte en exempta les marchands, membres de la 'ghilde' marchande, habitant cette ville, en même temps qu'il les exempta du tonlieu à Gravelines ${ }^{51}$. Ce dernier, cité dès 1107 à l'occasion de la donation par le comte de Flandre d'une rente de harengs, assignée sur ce tonlieu, à l'abbaye de Bourbourg, était sans doute le moins important des deux tonlieux ${ }^{52}$. Gravelines était visiblement avant tout un port de pêche sur l'estran de l'embouchure de l'Aa, dont la communication par eau avec Saint-Omer ne devint possible ou plus facile pour des bateaux plus grands que lorsqu'en 1163 la fondation de la ville de Gravelines marqua une étappe importante dans la canalisation de l'Aa ${ }^{53}$.

L'importance plus grande de Dixmude vers la même époque ressort également et avant tout du fait que le tarif de son tonlieu, qui reste malheureusement inconnu, a servi de modèle à celui de «Littersuerua» sur le Zwin, lorsque le comte Thierry d'Alsace convoqua les "échevins de son comté " et «principalement ceux de Dixmude » pour établir le tarif de celui-ci. Nous reviendrons plus loin sur cet aspect intéressant de la technique de décision. Il faut cependant noter dès à présent qu'à cette occasion le comte Thierry, dans la charte qui nous occupe, ne se réfère pas au tonlieu de Nieuport. Le tarif de celui-ci a cependant été mis par écrit en 1163 dans une charte émanant de son fils Philippe, dans laquelle celui-ci arrêta en même temps les dispositions régissant dorénavant le gouvernement et la justice de la

${ }^{49}$ A. Verhulst, leper in de lle en 12 e eeun; dans : Feestbundel C. Dekker; Utrecht, 1997.

50 H. VAN WERVEKE, "Hansa" in Vlaanderen en aangrenzende gebieden, Handelingen van het Genootschap «Société d'Emulation » TE Brugge, 90, 1953, p. 13 ; réimpression dans ID., Miscellanea Mediaevalia, Gand, 1968, p. 65.

${ }^{2}$ F. Vercauteren, Actes des comtes de Flandre 1071-1128, Bruxelles, 1938, n"127, p. 295.

s"Ibidem, n" 36, pp. 102-103.

"DERVILLE, Saint-Omer, pp. 69-73. 
ville fondée à cette même occasion ${ }^{54}$. On pourrait interpréter le fait que la charte de Thierry d'Alsace concernant «Littersuerua» ne se réfère pas comme modèle au tarif du tonlieu de Nieuport, mais à celui de Dixmude, que Nieuport et son tonlieu n'existaient pas encore lorsque la charte fut donnée. Celle-ci pourrait donc avoir comme terminus ante quem l'année 1163. Comme le tarif du tonlieu de Nieuport ressemble à certains égards à celui de "Littersuerua", on pourrait même supposer que le tonlieu de Dixmude, modèle de celui de «Littersuerua », aurait également servi de modèle à celui de Nieuport. Du point de vue de la diplomatique il n'est malheureusement pas possible de confirmer ce terminus ante quem, bien que le préambule de la charte qui nous occupe s'apparente très fort à ceux des chartes de la chancellerie des comtes de Flandre entre 1146 et $1166^{55}$.

Le terminus a quo est également difficile à déterminer, bien que l'époque du règne de Thierry commençant avec la régence de son fils Philippe en 1157 et l'association de celui-ci au pouvoir en 1163, inaugure, après le retour de Thierry de son troisième voyage en Palestine en août 1159 , une série d'initiatives comtales dans le domaine économique, dont la mieux connue - la fondation des villes portuaires nouvelles de Gravelines et Nieuport en 1163 - cadre bien avec l'émergence, vers la même époque ou peu de temps auparavant, du port de «Littersuerua» sur le Zwin et l'établissement du tarif de son tonlieu par le comte ${ }^{s t}$. Nous oserions donc proposer les années 1159-1163 ou alors, mais moins probablement vu l'activité minimale du comte Thierry après son retour de Terre Sainte en février 1166, la période

" DE Hemptine-Verhulst. De oorkonden der graven van Vlaanderen. II, 1, n" 222, pp. 344-348. Voir les travaux cités plus haut note 22.

"Le préambule "Quoniam generatio preterit et generatio succedit, memoriam vero rerum gestarum optime conseriant monumenta litterarum » est à rapprocher du texte de sept chartes comtales pour différents destinataires de 1146 à 1166. Dans sa thèse de doctorat inédite Thérèse de Hemptinne a attribué ce type de préambule à la chancellerie comtale de l'époque. Elle a même émis l'hypothèse que le rédacteur du formulaire de ce groupe d'actes aurait été un personnage de la région d'Ypres, attaché à la chancellerie comtale ; la plupart, mais pas tous, des destinataires concernés étant des institutions Yproises. Cette constatation n'est peut-être pas sans importance, vu que le tarif de «Littersuerua» réfère explicitement à celui de Dixmude, avant-port d'Ypres. Les sept chartes au préambulc quasi identique à celui de la mise par écrit du tarif de "Littersuerua» sont les suivantes: Th. DE HEMPTINNE - A. VERHULST, De oorkonden der graven van Vlaanderen, II, 1, n" 91, 125, 157, 174, 225, 256 et 266 . Il est à remarquer que les numéros 225 (1163) et 256 (18 avril 1166) sont des chartes du comte Philippe, tandis que le numéro 266 (21 décembre 1166) est émise par Thierry et Philippe ensemble.

". Voir les travaux cités plus haut note 22. 
entre février 1166 et le 17 janvier 1168 , comme date de cette initiative, provoquée cependant, comme le rappelle la charte de Thierry, par une plainte des marchands de Cologne ${ }^{57}$.

Or, la présence de ces derniers sur les rives du Zwin vers cette époque est d'une importance capitale pour l'histoire commerciale du comté de Flandre, d'autant plus qu'elle était inconnue jusqu'à présent. C'est également une donnée nouvelle dans la mesure où cette présence, pour la plupart des spécialistes qui s'en sont occupés, se situait plutôt vers la fin du XII et au début du XIII ${ }^{c}$ siècle ${ }^{5 x}$. Cette date tardive, qui a même étonné certains de ces auteurs, s'explique, selon eux, par ce qu'on est convenu d'appeler le commerce «actif» des Flamands, c'est-à-dire l'activité commerciale de marchands flamands à l'étranger, au détriment des marchands des régions visitées par eux. Cette concurrence a été particulièrement vive et parfois même source de conflits à propos du commerce flamand vers la Rhénanie et sur le Rhin lui-même. Dans ce contexte le conflit entre Gand et Cologne est célèbre ${ }^{59}$. Selon les meilleurs spécialistes de la question, ce conflit aurait débuté bien avant l'année 1169 , lorsqu'il est pour la première fois évoqué dans un document écrit. Il n'est donc pas impossible qu'il $y$ ait eu un lien entre ce conflit et la plainte vers 1159-1163/1167 des marchands de Cologne à propos du tonlieu du Zwin à «Littersuerua ». La cause en était probablement l'ascension rapide de Cologne, dont les marchands, privilégiés à Londres en $1157^{\circ}$, auraient à partir des années cinquante du XII ${ }^{\mathrm{c}}$ siècle, commencé à concurrencer le commerce « actif » des Flamands. Jusque là ceux-ci n'auraient guère rencontré d'obstacles ou de concurrence lors de leurs voyages à l'étranger, d'où ils amenaient vin, laine, métaux et autres matières premières dont la Flandre avait besoin. On en vient même à se demander si cette idée de Van Werveke d'un commerce «actif» des Flamands, sans concurrence étrangère jusque vers 1150 et concurrencé par des mar-

"7. Pour un exposé sur les motifs précis de la datation proposée, nous renvoyons à l'édition en annexe.

${ }^{9} \mathrm{H}$. VAN WERVEKE, Der flandrische Eigenhandel im Mittelalter, HANSISCHE GeschichtSBLÄtTER, 61, 1936, p. 17, réimpression dans Id., Miscellanea Mediaevalia, p. 52 ; F. Blockmans, Het Gentsche Stadspatriciaat tot omstreeks 1302, Anvers-La Haye, 1938, p. 182 ; Ph. Dollinger, La Hanse, Paris, 1964, p. 58.

"Voir les études de VAN WerveKe et Blockmans citées à la note précédente et en dernier lieu F. IRSIGLER, Köln und die Staufer im letzten Drittel des 12. Jahrhunderts, dans W. Hartmann (éd.), Europas Städte zwischen Zwang und Freiheit, Regensburg, 1995, pp. 83-96.

(x) E. ENNEN, Kölner Wirtschaft im Früh- und Hochmittelalter, dans H. KelLENBENZ (éd.), Zwei Jahrtausende Kölner Wirtschaft. 1, Cologne. 1975, p. 143. 
chands étrangers depuis lors jusqu'à la fin du XIII ${ }^{\circ}$ siècle, lorsque les Flamands auraient renoncé à leurs voyages à l'étranger ${ }^{61}$, puisse être maintenue. Ne vaudrait-il pas mieux admettre l'existence de deux réseaux parallèles "actifs », aussi bien celui de Flamands à l'étranger que celui de marchands étrangers en Flandre, puisque au début du XII ${ }^{*}$ siècle des marchands italiens étaient présents en mars 1127 à la foire d'Ypres ${ }^{62}$ et puisqu'encore au XIV ${ }^{\mathfrak{c}}$ siècle des marchands flamands opéraient en Angleterre ${ }^{63}$.

Que la plainte des marchands de Cologne au sujet du tonlieu de "Littersuerua» ne fut pas un fait isolé, mais qu'il cadre bien dans un conflit commercial plus large entre le comté de Flandre et la ville de Cologne vers cette époque, semble trouver une confirmation dans le fait que le comte Thierry convoqua, d'après la charte qui nous occupe, les " échevins de son pays" ("convocatis itaque scabinis terre mee») et «plus particulièrement ceux de Dixmude» («et illis precipue de Dicsmuda») pour examiner la plainte des marchands de Cologne. S'il n'y avait pas eu, vers la même époque, les difficultés entre Cologne et Gand, on aurait pu traduire et interpréter les mots "terre mee » par « châtellenie du Franc de Bruges », la circonscription administrative et judiciaire dont faisaient partie à la fois "Littersuerua» et Dixmude. Jusque vers l'époque qui nous occupe et encore en 1163, les exemples d'une convocation formelle par le comte de tous les échevins explicitement désignés comme étant « de la Flandre » se rapportent, en effet, au Franc de Bruges ${ }^{\omega}$. Mais précisément à partir de la même époque, le comte Philippe d'Alsace, associé au gouvernement de son père Thierry depuis 1163, aurait, selon F. L. Ganshof, organisé la consultation des échevins des sept grandes villes flamandes (Gand, Bruges, Ypres, Saint-Omer, Arras, Lille et Douai) en convoquant les «scabini Flandriae », bien qu'un spécialiste de l'histoire des institutions représentatives, feu Jan Dhondt, ne signale la première preuve écrite de l'usage de l'expression «scabini Flandriae» qu'en $1241^{6.5}$. Mais

"VAN Werveke, Flandrische Eigenhandel, pp. 45-59 de la réimpression.

: $:$ Galbert de Bruges, chap. 16, trad. J B. Ross, Galbert of Bruges. The Murder of Charles the Good, count of Flanders, New York, 1967², pp. 123-124, n. 20.

${ }^{\circ} \mathrm{M}$. DE LAET, De Vlaamse aktieve handel op Engeland in de eerste helft van de XIVe eeun, ECONOMISCHE GESCHIEdENIS VAN BELgIË. HANDELINGEN VAN HET Colloquium te Brussel 1971, Brussel, Algemeen Rijksarchief, 1972, pp. 223-231.

${ }^{4} \mathrm{E}$. WARLOP, De vorming van de grote schepenbank van het Brugse Vrije (lle13e eeuw), Standen En LANDEN, 44, 1968, p. 5.

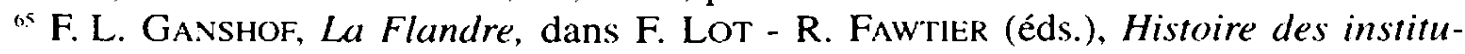
tions françaises au moyen âge. 1, Paris, 1957, pp. 375-376; J. DHondt, Les origines des Etats de Flandre. STanden en Landen - Anciens Pays et Assemblées d'Etats, 1 , 
comme l'a fait remarquer un autre spécialiste des institutions représentatives, W. Prevenier, le titre officiel de «scabini Flandriae» des représentants des grandes villes flamandes en 1241 n'a de fait rien

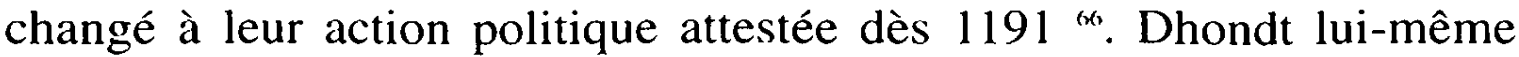
admet d'ailleurs que la procédure de consultation des échevins des sept grandes villes, utilisée surtout en matière de politique économique, pourrait remonter à la fin du $\mathrm{XII}^{\mathrm{c}}$ ou au début du XIII ${ }^{\circ}$ siècle. La pratique elle-même d'une action collective des grandes villes flamandes, sans être désignée explicitement comme réunion des «scabini Flandriae », pourrait même, de l'avis de Prevenier, remonter, du moins sur le plan politique, aux évènements de 1127-1128. Puisque la présence de marchands de Cologne en Flandre peut être reculée dans le temps jusqu'au milieu du XII' siècle, il n'est nullement exclu que la charte qui nous occupe soit le premier exemple écrit d'une technique de décision en matière de politique étrangère, économique, commerciale et monétaire, caractérisée par la participation des grandes villes du comté, inaugurée au cours des évènements de 1127-28 et qui se maintiendra pendant le reste du moyen âge.

En ce qui concerne la participation de Dixmude dans le cas qui nous occupe - une ville dont l'importance ne peut être comparée à celle d'aucune des grandes villes du comté citées ci-devant - nous avons déjà proposé plus haut de l'expliquer par le fait que jusqu'à la formation du Zwin à partir de 1134 et l'émergence, à peu de distance de Bruges, de ports de marée sur ses rives, Dixmude était, à part le port de pêche de Gravelines à l'embouchure de l'Aa, le seul port de mer du comté facilement accessible comme avant-port d'une grande

1950, pp. 3-52, réimpression dans W. Blockmans (éd.), Estates or Powers. Essars in the parliamentary history of the Southern Netherlands by Jan Dhondt, Heule, 1977 (Standen en Landen - Anciens Pays et Assemblées d’Etats, 69), pp. 73-74. Le terme «scabini terre mee » (dans le sens de «tous les bancs scabinaux du territoire comtal») se rencontre pour la première fois dans la «keure » de Guillaume Cliton pour SaintOmer de 1127 (article [1] «ipsisque scabinis libertatem, qualem melius habent scabini terre mee »), et ensuite dans les «keures» (de Thierry et de Philippe d'Alsace, de Baudouin IX et Marie de Champagne) pour cette ville. G. Espinas, Recueil de documents relatifs à l'histoire du droit municipal en France des origines à la Révolution. Artois, I, Paris, 1934, n" 622-628. Pour la "keure 》 de Guillaume Cliton, voir R. C. Van Caenegem, De keure van Sint-Omaars van 1/27, dans Revue. D'Histoire du DROIT, L, 1982, pp. 253-262, où sont signalées les autres éditions (pp. 255-256, notes 13 et 19); il faut y ajouter pour les «keures » de Thierry et de Philippe d'Alsace Th. DE HEMPTINNF, - A. Verhul.st, De oorkonden der graven van Vlaanderen, II, 1, n" 2, 231 et 233. Le terme "scabini terre mee », utilisé ici dans l'exposé de la charte du comte Thierry, n'apparaît pas dans d'autres chartes comtales du XII' siècle.

* W. Prevenif.R, De Leden en de Staten van Vlaanderen. (1384-1405). Bruxelles, 1961, pp. 32-33. 
ville, en l'occurrence Ypres. La charte de Thierry d'Alsace concernant le tonlieu de "Littersuerua » dit plus particulièrement que dorénavant ce tonlieu comtal sera levé avec fermeté selon les modalités et les quantités prescrites pour le tonlieu de Dixmude, que les échevins convoqués et ceux de Dixmude en particulier ont fait connaître en toute vérité à cette occasion ( « secundum formam et quantitatem thelonii in Dicsmuda, nostrum et(iam) theloneum Letraswerf, consulentibus scabinis et illis de Dicsmuda, veritatem eius rei proferentibus, firmiter coequavi »).

Malheureusement aucun tarif de tonlieu de Dixmude n'est conservé, du moins à notre connaissance, certainement pas parmi les archives de l'administration financière du comté de Flandre ${ }^{67}$. La cause en est probablement l'abandon par le comte de l'exploitation directe du tonlieu et sa concession au seigneur de la ville pour une somme forfaitaire qui, en 1187 , se montait à 60 livres dans le plus ancien compte général conservé du comté, connu sous le nom de 'Gros Brief' ' '. A cause de son caractère forfaitaire le montant de cette somme ne peut être utilisé directement comme indication de l'importance du tonlieu, bien qu'il soit le plus haut de ceux mentionnés dans le 'Gros Brief', suivi par Biervliet avec $57 \mathrm{lb}$., ici en exploitation directe («hoc anno»), par Courtrai avec une somme forfaitaire de $34 \mathrm{lb}$. et par d'autres villes, parfois plus importantes, avec le plus souvent des sommes forfaitaires toutefois moindres, comme Lille et Douai avec $15 \mathrm{lb}$., contre de petites villes comme Bailleul et Furnes avec des sommes plus importantes, respectivement de 30 et $26 \mathrm{lb}^{69}$. L'abandon par le comte de l'exploitation directe du tonlieu de Dixmude a peut-être suivi de peu l'établissement d'un tonlieu à Nieuport en 1163, qui lui, tout comme la plupart des autres tonlieux comtaux importants, connus avec leur rendement au XIIIe siècle, par exemple Damme, ne figure pas dans le 'Gros Brief' de 1187, mais dans le compte du domaine «nouveau » du comte, appelé 'renenghelle' et dont les premiers exemplaires conservés datent de la deuxième moitié du XIII' siècle ${ }^{70}$. Dans le 'Gros Brief', en effet, ne

${ }^{17}$ R. H. BALTIER - J. SORNAY, Les sources de l'histoire économique et sociale du moyen âge. Les états de la maison de Bourgogne. 1. Archives des principautés territoriales. 2. Les principautés du Nord, Paris, 1984, pp. 124-138.

is Verhulst - Gysseling, Compte Général, pp. 126, 142.

${ }^{6}$ Ibidem, p. 126, n. 2.

7" R. MONIER, Les institutions financières du comté de Flandre, Paris, 1948, p. 87 ; LUYKX, Grafelijke financiële bestuursinstellingen, pp. 48-52. G. DECLERCQ, Kamer van de Hoofdredeninge (12de eeun-1795), dans W. PREVENIER en B. Augustyn, De gewestelijke en lokale overheidsinstellingen in Vlaanderen tot 1795 [Algemeen Rijksarchief en Rijksarchief in de provinciën, Studia, 72], Bruxelles, 1997, pp. 171-187. 
furent enregistrés, pour la plupart, des revenus et des dépenses qui, par suite de concessions et d'assignations perpétuelles ou à long terme, à cens ou à bail, étaient déjà devenus immuables en $1187^{\prime \prime}$. Ce n'était donc pas le cas de «Littersuerua», dont le tonlieu ne figure pas dans le 'Gros Brief' de 1187, ni d'ailleurs dans la 'renenghelle' très complète de 1296, où figure son successeur, le tonlieu de Damme ${ }^{72}$.

A défaut du tarif du tonlieu de Dixmude, l'analyse de celui de "Littersuerua » doit se faire de préférence en le comparant à celui de Nieuport, qui lui est contemporain (1163), et naturellement aussi avec celui de Damme, qui date de $1252^{73}$. La première chose qui ressort d'une telle comparaison c'est que le tarif de Damme est beaucoup plus long que les deux autres. Pendant la période de presqu'un siècle qui s'est écoulée depuis la mise par écrit de ces derniers, la liste des produits taxés à «Littersuerua »-Damme a dû s'allonger, notamment parce que Damme, devenu entretemps l'avant-port de Bruges, était déjà au milieu du XIII' siècle l'un des plus grands ports de l'Occident. Malgré cette évolution et malgré le fait que la structure d'un tarif de tonlieu soit généralement très anarchique, quelques ressemblances et analogies entre le tarif de Damme et son précurseur de "Littersuerua ", plutôt qu'entre ce dernier et le tarif contemporain de Nieuport, se laissent encore déceler. Ainsi en est-il du début des deux tarifs, de «Littersuerua » et de Damme, qui commencent chacun, de façon assez logique, par des dispositions générales concernant les taxes auxquelles sont soumis les différents types de navire. Bien que les mêmes types de navire, tant à «Littersuerua» qu'à Damme, se retrouvent, avec les mêmes taxes, dans le tarif de Nieuport, leur place dans ce dernier n'est pas au début ${ }^{74}$. Il n'y a d'ailleurs pas ou peu d'ordre dans la liste des produits de ce dernier tarif, alors que le début du tarif de Damme, c'est à dire une bonne quinzaine de produits ou groupes de produits, ressemble assez bien, quant à l'ordre d'énumération, au tarif de «Littersuerua » dans sa totalité. Il y a évidemment des différences apparentes pour lesquelles une explication peut néanmoins

"Verhulst - Gysseling, Compte Général, pp. 137-138; A. Verhulst - B. Lyon, Medieval Finance. A Comparison of Financial Institutions in Northwestern Europe, Bruges-Providence, 1967, pp. 29-35.

72 MONIER, Institutions financières, p. 87.

${ }^{71}$ Voir plus haut, respectivement notes 54 et 35.

${ }^{74}$ Sur ces types de navire voir l'étude de R. Degryse, De maritieme aspecten van de keure van Nieuwpoort van 1/63, Mededel.jngen VAN DE MARINe ACAdemie, 20, 1968, pp. 63-82, basée sur une comparaison des tarifs de tonlieu de Nieuport et Damme, le tarif du tonlieu de 'Littersuerua' étant resté inconnu à l'auteur. 
être trouvée : c'est le cas du vin, qui vient en tête de liste à Damme en 1252 , alors que dans le tarif de «Littersuerua » le vin, avec le miel, la bière, la poix, la cendre et l'hydromel, se trouve quelque part au milieu de la liste. Est-ce que cette différence s'explique par le fait qu'au milieu du XII' siècle les marchands flamands, notamment de Gand, allaient encore chercher eux-mêmes le vin en Rhénanie ${ }^{75}$, alors qu'au milieu du XIII" siècle Damme était déjà devenu le premier port pour l'importation des vins du Poitou, devenus plus importants entretemps que les vins du Rhin ${ }^{76}$ ?

Dans les trois tarifs, laine, fils et draps se trouvent, après le poisson à "Littersuerua » et après le vin à Damme, pratiquement en tête de liste, ce qui n'étonnera guère. Cette fois la ressemblance est la plus forte entre les tarifs de "Littersuerua " et de Nieuport, aussi bien en ce qui concerne le montant des taxes que du point de vue de la nature des matières importées. Alors qu'un siècle plus tard à Damme, où la liste des produits textiles en 1252 est en même temps beaucoup plus diversifiée, les draps forment la majorité, à «Littersuerua» au XII' siècle, où apparaissent deux sortes de draps et surtout à Nieuport, où l'on n'importe en 1163 que de la laine brute et des fils, notamment d'Angleterre et très probablement destinés à Ypres, et pas de draps, la matière première domine largement.

A cet égard les trois tarifs reflètent de façon suggestive la structure économique de la Flandre en ce qui concerne l'industrie textile et son évolution du XII' au XIII' siècle: rôle de l'importation de la matière première, notamment d'Angleterre et prépondérance d'Ypres comme centre de production au XII ${ }^{\circ}$ siècle, avec un début de Bruges comme centre de consommation de produits finis, devenant au XIII' siècle un marché international ${ }^{77}$.

Après quelques produits comme le cuir et la graisse, les métaux occupent dans les trois tarifs sous considération la deuxième ou la troisième place, non seulement dans l'ordre d'énumération, mais aussi du point de vue de leur importance et surtout de leur diversité, tant des matières premières (fer, cuivre, plomb, acier, étain) que des produits finis. Alors qu'à Nieuport en 1163 on n'importait que les principales matières premières, la liste de «Littersuerua » frappe par la grande diversité de produits métalliques qu'on y importait, vraisemblablement

"Blockmans, Stadspatriciaat, pp. 177-180.

"PIRENNE, Un grand commerce, pp. 599-601.

"H. VAN Werveke, Esquisse d'une histoire de la draperie, dans G. DF. Pofrck, La draperie médiévale en Flandre et en Artois. I. Technique et terminologie. Bruges, 1951, pp. 7-25 ; réimpression dans VAN WERVEKE, Miscellanea Mediaevalia. pp. 350-364. 
en premier lieu d'Allemagne et notamment de Cologne. Cette différence suggère que le tarif de "Littersuerua » constitue probablement, vis-à-vis de son modèle de Dixmude que nous ne connaissons malheureusement pas, un stade plus évolué. C'est sans doute de Cologne, où elles étaient fabriquées en grand nombre, qu'arrivaient à "Littersuerua» peu après le milieu du XII" siècle déjà, les armes ( "gladii », «hastae») qu'on retrouvera à Damme en $1252^{7 *}$. Leur importation à «Littersuerua » confirme donc indirectement la présence de marchands de Cologne sur les bords du Zwin peu après le milieu du XII' siècle et l'importance que les autorités du comté et des villes flamandes ont attachée à leur plainte, qui est à l'origine de la mise par écrit du tarif du tonlieu de «Littersuerua» dans la charte de Thierry d'Alsace que nous venons d'analyser.

${ }^{*}$ ENNFN, Kölner Wirtschaft, pp. 137-138. 
Thierry, comte de Flandre, suite à une plainte des bourgeois de Cologne et après concertation avec ses scabini terre et plus particulièrement ceux de Dixmude, institue un tarif de tonlieu pour Littersuerua, pareil à celui de Dixmude.

\author{
S.I. ; s. d. [16 août 1159-janvier 1164 , \\ ou, moins probablement, février 1166-17 janvier 1168].
}

A. Original. : présumé perdu.

[B. Cople ol DOUBL.F DF l'original (?) d'avant 1269, attaché à une charte de Marguerite. comtesse de Flandre et de Hainaut, et Guy, comte de Flandre et marquis de Namur, du 19 septembre 1269, d'après $A($ ?) ; présumé perdu]. - C. COPIE s. XV, sur deux feuilles de papier cousues ensemble, d'après B. A.D.N., Lille, B 1321/1599-1600. Au dos: 1) de la même main que la copie : 'Copie comment on doit prenre et recevoir le tonlieu de Graveling(hes), tant par mer comme par terre et quantbienn chacune neif doit. IIII ${ }^{\mathrm{XX}} \mathrm{XXX}$ '. 2) main s. XV : 'littere sunt appense'.- 3) main s. XVIII : 'Gravelines thonlieu 1269. [recouvert]n septembre 1269’. - 4) main s. XIX: '1269. septembre, le jeudi après l'Exaltation de Sainte Croix'.

NOTE CONCERNANT LA DATATION : La datation que nous proposons ici et plus particulièrement les termes a quo (retour du comte Thierry de son troisième voyage en Terre Sainte) et ante quem (départ de Thierry pour son quatrième voyage en Terre Sainte), ou, moins probablement, retour du comte de son quatrième voyage et décès ', est motivée par différentes considérations de fond et de forme.

En ce qui concerne le fond il faut, comme il a été exposé dans l'introduction, prendre en compte la mention de la plainte des marchands de Cologne, l'époque des initiatives comtales en matière économique, la chronologie des voyages du comte Thierry en Orient et le fait qu'il n'est pas fait référence au tarif du tonlieu d'un autre nouveau port de mer, Nieuport (1163). mais à celui de Dixmude, certainement plus ancien que ce dernier. Tous ces éléments semblent indiquer la période qui commence au retour en Flandre du comte Thierry en 1159 et se termine avec son demier départ en janvier 1164 . Cette période, la dernière pendant laquelle Thierry tient encore solidement en

' Concernant les dates des troisième et quatrième voyages du comte Thierry en Terre Sainte et sa biographie, voir: H. VAN WERveke, Filips van de Elzas en Willem van Tryus, dans Mededelingen van de Koninklijke Vlaamse academie voor Wetenschappen, Letteren en Schone Kunsten van België. Klasse der Letteren, XXXIII, 1971, nr. 2, appendix 2, pp. 30-31, et Th. DE HEMPTINNE, "Biographie de Thierry d'Alsace, comte de Flandre (1128-1168)", dans Annales de l'Est. 5" séric, 43" année, numéro 2, 1991 , pp. 83-108. 
main les rênes du pouvoir, celle aussi qui voit l'influence grandissante du conseiller Robert d'Aire à la cour, est sans doute la plus logique pour situer ce document. Il est toutefois étonnant que le comte Philippe n`ait pas été associé à cette initiative, alors qu'il assiste déjà souvent son père dans des décisions importantes et émet des actes seul, même de la plus haute signification politique et économique, en son propre nom. Pour expliquer l'absence du jeune comte dans la formule de suscription d'un document de cette importance à cette époque, il faut sans doute admettre que la plainte était adressée au comte Thierry seul ou que le formulaire du protocole a été repris littéralement au modèle, le tarif de Dixmude. L'inconvénient de l'absence de Philippe dans la suscription est encore plus flagrant, si on prend en considération une datation plus récente, c'est-à-dire la période entre le retour de Thierry en 1166 et son décès en 1168. En effet, le vieux comte est, à quelques exceptions près, pratiquement absent des suscriptions des chartes comtales datées de cette époque. En outre, l'argument concernant la référence au tarif, non de Nieuport qui n'aurait pas encore existé, mais de Dixmude, tombe, si on accepte cette datation plus récente.

Du point de vue de la forme, outre la suscription, scul le préambule nous donne une indication chronologique. Il s'agit d'une formule de chancellerie en vogue entre 1146 et 1166 , employée dans des chartes émanées du comte Thierry, mais aussi de son fils. Cette constatation confirme notre datation hypothétique basée sur des arguments de fond, mais laisse posée la question d'une éventuelle reprise du formulaire à un modèle inconnu, celui du tarif de Dixmude.

Quoniam generatio preterit et generatio succedit, memoriam vero rerum gestarum optime conservant monumenta litterarum, ego Th[eodericus] " Flandrens(ium) comes, communi consulens utilitati, ut o(mn)em iniquam et extortam exactionem a iure nostro excl(u)derem, ea que ne de theloneo portus de Letraswerf instituta sunt scripto commendare curavi. Convocatis itaque scabinis terre mee et illis precipue de Dixsmuda, propter querimoniam civium Coloniens(ium) qui se aggravari in Letraswerf " per iniustam exactionem asserebant, secundum formam et quantitatem thelonii in Dicsmuda nostrum etiam "theloneum Letraswerf ", consulentibus scabinis et illis de Dicsmuda ", veritatem eius rei $\mathrm{p}$ (ro)ferentibus, firmiter coequavi.

Prima est institutio navibus magnis et parvis ', secundum quantitates earum: magne naves $q(u e)$ dicuntur cnorbusa, snac et om(ne)s turbeate" dab(un)t

"Th[eodoricus] Thomas (sic) C. - "Letraswerf] le Traswerf (sic) C. "etiaml et (sic) C. - "Dicsmuda] Dicsnuda $C$. - turbeate (sic) CJ trabeata dans le tarif du tonlieu de Damme de 1552, voir ci-dessus, note 35.

'Sur ces types de navires, cités ici et plus loin dans le texte et dont les mêmes noms en langue vulgaire se retrouvent dans le tarif de Nieuport de 1163 (éd. DE HEMPTINNEVERHULST, n" 222 ; voir plus haut, notes 22 et 54) et dans celui de Damme de 1252 (éd. Höhlbaum, n" 432 ; voir plus haut, note 35 ), voir R. DEGRYSE, « De maritieme aspecten van de keure van Nieuwpoort van 1163 ", Mededelingen van de Marine Academie, 20 (1968), pp. 63-82. 
XII d.; minores vero, videlicet kogan et om(ne)s cum losabo(in)ga dab(un)t VIII d. ; envare(n) dab(unt) quatuor d., scuta cum bort dab(it) II d., scuta absque bort I d.; et $\mathrm{h}(\mathrm{ec})$ si on(er)ate vener(int), ex hiis navibus, qu(e)cumque annonam vel fabam, pisam vel salem, rapas vel poma vel nuces attulerit, de quinquaginta raseriis dab(it) $s$ (emis) raser(ie), si plus, quantumcumque fuerit, dab(it) I r(aseriam), si minus, ex XXV r(aseriis) quart(am) partem r(aserie), si minus quam $X X V$, nichil.

Si vero navis propter alleviationem sive in terram sive in aliam navem res suas posuer(it) om(ne)s, nichil inde dab(it); si vero extra navem annonam vel cetera supradicta vendiderit de val(e)nte marcham venditor dab(it) VI d., et emptor, si in aliam navem posuerit et ita abduxer(it), nichil inde, nisi navis theloneum sicut supradictum est, dab(it); si vero plaustris abduxerit, de plaustro dab(it) IIII d.

Quicumque alec salsum ' ahduxerit et in navi vendiderit nichil, nisi navis ut supradictum est, dab(it) theloneum; si extra navem vendiderit, uterque, tam emptor quam venditor, dab(it) de mill(enari)o I d. Qui alec recens attulerit, sive in navi sive extra navem vendiderit, uterque, tam emptor quam venditor, de mill(enari)o I d.

Quicumque salmones adduxerit, si in navi vendiderit, nichil, nisi navis theloneum, inde dab(it) ; si extra vendiderit, tam emptor quam venditor dab(it) de C, IIII d.; emptor vero, sive in navi sive extra navem emerit, dab(it) de $C$, IIII d. Si makeuis adduxerit et in navi vendiderit nichil, nisi navis theloneum, dab(it) ; si vero extra vendiderit, dab(it) de mill(enari)o, uterque II d.

Qui lanam attulerit et in navi vendiderit nichil, nisi navis thel(oneum), inde dab(it), et e[xtra]' navem vendiderit, de pisa lane dab(it) Il d., et si abduxerit, de pisa dab(it) I d. ; et emptor, sive intus sive extra emerit, dab(it) de pisa lane II d.

Quicumque caseos vel roet vel smera vel spec q(uod) ponderatur et in navi vendiderit, nichil, nisi navis ius, dabit, et si extra navem vendiderit, et emptor et venditor dab(it) de pisa II d., et si abduxerit, de pisa dabit I d., exceptis caseis Flandrensibus de quorum, si pisa venditur, datur ab utroque $I \mathrm{~d}$. et de bacone s(emis) d.

Qui plumbum in [na]vi " vendiderit, nichil, nisi navis thel(oneum), [da]b(it) ", et si extra vendiderit, venditor dab(it) de pisa I d., et emptor, sive in navi sive extra navem emerit, de pisa $I \mathrm{~d}$.

' extra] manque $C$, à cause d'un trou dans le papier: - " navil na manque $C$, à cause d'un trou dans le papier: - "dabit] da manque C. à cause d'un trou dans le papier.

\footnotetext{
'Sur les différents types de poissons de mer: même remarque qu'à la note précédente.
} 
Qui stannum ' in navi vendiderit, nichil, nisi navis thel(oneum), inde dab(it), et si abduxerit, de mill(enari)o dab(it) IIII d., et si extra vendiderit, dab(it) de val(e)nte marcham, VI d. ; et emptor, sive in navi sive extra navem emerit, dab(it) de val(e)nte marcham, VI d.

Qui cuprum adduxerit, ubicumque abd[u]xerit ', dab(it) de plaustrato VI d., et si vendiderit, uterque, et venditor et emptor, dab(it) de val(e)nte marcham, VI d.

Qui ceram adduxerit et abduxerit, si sit pisa cere vel plus, dab(it) IIII d., et si vendiderit, uterque dab(it) de val(e)nte marcham VI d.

De sarcina $q(u e)$ dicitur pac cum cordis IIII d.; de pivo cum spic II d.; de sista serata, de qualibet re plena, IIII d.; de sista vacua, si valuerit V s., dab(it) I d., si peior, nichil.

De gladiis, scutellis, tin(n)is, situlis, qui simul valent(es) V s. vendiderit, dab(it) I d., si minus, nichil, et si abduxerit nichil inde dab(it); de XXV hastis I d.

Qui plaustrum ferri abduxerit, dab(it) IIII d. ; de scuta II d. ; si vendiderit de val(e)nte marcham, VI d., et emptor similiter, et de stael similiter.

De $t u n(n) a$ vini uterque, et emptor et venditor, dab(it) IIII d.; de $t u n(n) a$ mellis, qui abduxerit, dab(it) IIII d., si vendiderit, dab(it) de val(e)nte marcham, VI d., et emptor similiter; de $\operatorname{tun}(n) a$ servisie I d. ; de $\operatorname{tun}(n) a$ p[ici]s * IIII d. ; de $\operatorname{tun}(n) a$ ciner(is) II d. ; de $\operatorname{tun}(n) a$ medonis I d.

De culcitra uterque II d. ; de plumari ' uterque I d.

De equo uterque II d. ; de vacca uterque I $\mathrm{d}$.; de porco vel ove uterque s(emis) d.

De plaustrato ligni I d., de carra s(emis) d. ; plaustrum cum fimibus I d., plaustrum annone I d., plaustratum cuiuslibet fructus I d. et carra s(emis) d. ; si transier(it) et nichil vendiderit, nichil dab(it).

Qui vendiderit VI ${ }^{\mathrm{I} u m}$ partem ponderis fili, dab(it) s(emis) d., et qui emerit valens $V$ s., dab(it) I d.

De panno albo laneo integro II d.; de panno colorato uterque dab(it) de val(e)nte marcham, VI d.

Nemo de vestibus, qu(a)s ad usus proprios aut familie sue emerit, thel(oneum) dab(it).

Ex uno gherut quar(ele)n s(emis) d. ; ex uno gerud mol(arum) I d. ; ex uno sliipsteen I d. ; ex uno fardeel wedarum II d. ; ex uno last quar(ele)n IIII d. ; ex uno last mol(arum) IIII d. ; ex uno pac caldar(iarum), patellarum et pelvium et messinc, si abduxerit, dab(it) IIII d., et si vendiderit, uterque de val(e)nt(e) "m marcham, dab(it) VI d.

' stannum] tm ou tin abrégé (sic) C. - 'abduxerit] u manque C. à cause d'un trou le' papier: - "picis] plicils trois lettres manquent $C$. à canse d'un trou dans le papier: 'plumaril pluinari (sic) C. - "val(e)n(te)l abrégé valm (sic) $C$. 
De C placit(is) I d., de graling(is) II d. ; de C buttis s(emis) d. ; de C scelleuis I d.

Ex coriis dacr(a) I d.

Scuta cum porro et allio I d. ; scuta cum olere I d. ; scuta cum turbonibus I d. ; plaustrum cum turbonibus IIII turbones.

Nulla navis de transitu aliquid dab(it).

De pomis et piris qu(e) conduct(ores) attulerint nichil dab(unt).

De om(n)i quod infra navem venditur nichil, nisi navis thelon(eum), debetur.

Quicumque partem rerum vendiderit suarum et partem reduxerit, de parte vendita thelon(eum) dab(it), de reliqua nichil.

Navis que portum intraverit et ibi mar(is) temperiem expectaverit, et nichil preter victum emerit, ab om(n)i thelon(eo) libera exibit.

Marguerite, comtesse de Flandre et de Hainaut, et Guy, comte de Flandre et marquis de Namur, son fils, informent leurs receveurs de tonlieu à Gravelines, qu'ils auront dorénavant à lever ce tonlieu selon le tarif annexé, établi par leur prédécesseur le comte Thierry lpour Littersuerua].

\section{S.l. ; 19 septembre 1269.}

A. ORIGINAL : présumé perdu.

B. COPIE s. XV, sur deux feuilles de papier cousues ensemble, d'après A (?). A.D.N.. Lillc. B 1321/1599-1600. Au dos: 1) de la même main que la copie: Copie comment on doit prenre et recevoir le tonlieu de Graveling(hes), tant par mer comme par tcre et quantbienn chacune neif doit. IIII ${ }^{\mathrm{xx}} \mathrm{XXX}$ '. - 2) main s. XV : 'littere sunt appense'. - 3) main $\mathrm{s}$. XVIII : 'Gravelines thonlieu 1269. [recouvert]n septembre 1269'. 4) main s. XIX : '1269, septembre. le jeudi après l'Exaltation de Sainte Croix".

Nos Margareta Flandrens(is) et Hann(oniensis) comitissa et Guido filius eius comes Flandr(ensis) et march(io) Namurcensis", notum facimus universis quod nos volumus quod om(ne)s receptor(es) thelonei de Graueli(n)g(is), tam in aqua quam in terra, consuetudines ac thelonea de cetero recipiant "secundum tenorem scripti per bon(e) memor(ie) Theodericum" quondam comitem

\footnotetext{
"Namurcensis] Nam(ur)enc() (sic) C. - " recipiant] recipiantur (sic) $C$.
} 
Fland(ri)e, predecessorem nostrum, confecti, cui hec nostre presentes littere sunt appense, et quod aliam monetam non exigant nec recipiant, nisi nostram monetam Fland(ri)e.

Ita tamen quod si alique m(er)candis(ie) ad predictum portum nostrum de Graueling(is) applicuerint, de quibus quantum solvi debeat in predicto scripto non fuerit determinatum, volumus quod in predictis $m$ (er)chandis(iis) consuetudines et thelonea recipiantur secundum valorem et quantitatem $m(e r) c h a n-$ dis(iarum) in predicto scripto contentarum, salvis nobis ibidem foragio nostro et denar(iis) qui dicuntur scalcpenninghe; quos denar(ios) recipi volumus secundum consuetudines in villa nostra de Graueling(is) hactenus habitas et obtentas et salvis nobis denariis duobus qui debentur pro quolibet dolio gaugiato.

Et sciendum est quod quicumque thelon(eum) nostrum in dicta villa absportaverit in forefacto sexaginta solidorum erit, de quibus due partes erunt nostre et tertia pars ville nostre de Graueling(is) predicte. nenda.

In quorum testimonium presentibus litteris sigilla nostra duximus appo-

Datum anno Domini millesimo CC" $\mathrm{LX}^{\prime \prime}$ nono, mensis septembris, die Jovis post Exaltationem Sancte Crucis.

- Theodericum] Theoderici cum (sic) $C$. 